\title{
Development of SCAR makers for longan (Dimocarpus longan L.) authentication in Vietnam
}

\author{
VIET The Ho $^{1^{*}}$, QUOC NGUYen NGo ${ }^{2}$ \\ ${ }^{1}$ Faculty of Biotechnology, Ho Chi Minh City University of Food Industry, Ho Chi Minh City, Vietnam \\ ${ }^{2}$ Faculty of Biology, University of Science, Ho Chi Minh City, Vietnam
}

\begin{abstract}
Longan (Dimocarpus longan L.) is an important fruit plant in Vietnam and therefore the demand of genetic conservation and variety authentication is of high importance. Due to the limitations of chemical and morphological longan characterization approaches, it is necessary to develop more precise DNA-based methods. In this study, we focused on developing SCAR (Sequence Characterized Amplified Region) markers for longan identification. The total of 11 longan accessions collected from different provinces in Vietnam were genetically characterized by using 20 RAPD (Random Amplified Polymorphic DNA) primers to identify polymorphic RAPD fragments. Twenty two polymorphic RAPD fragments were obtained and 2 of them were further sequenced and developed into SCAR markers. The sequences of specific RAPD fragments were used to design primers. Totally, 2 pairs of SCAR primers were archived. These primers showed significant effectiveness for cultivar identification of 2 favourite longan cultivars of Vietnam namely "Duong phen Hung Yen" and "Chin muon Hung Yen".
\end{abstract}

Key words: Longan, molecular markers, RAPD, SCAR

\section{Introduction}

Longan (Dimocarpus longan L.) is the most popular member of the Sapindaceae family which produces arillate fruits with sweet, translucent and juicy flesh. This fruits have high sugar and several vitamins and minerals such as vitamin C, thiamine, niacin, riboflavin, phosphorus, iron and calcium content. Longan pulp has long been used not only as a fruit but also in a traditional medicine for health promotion purposes like enhancing blood metabolism, nerve soothering, amnesia prevention, insomnia relief and longevity extension. This fruit can be eaten fresh or processed into different forms (frozen, canned, dried, juice, wine, pickles, preserves, ice-cream and yoghurt) (FAO, 2004).

In Vietnam, longan is one of important fruit trees with total cultivation area of 75200 ha, over $70 \%$ of longan production is located in Mekong Delta in the south of Vietnam (Wong, 2000). Vietnam is also the second biggest longan exporter in the world after Thailand, with annual export value of approximately 52 million USD (Tien Giang Promotion Agency, 2017). Despite its high economic importance, breeding and conserving of this plant species in Vietnam are still limited (FAO, 2004). Longan population is unsystematically classified, which is based on plant or fruit morphology. So far the studies of longan are scant, and mostly concerning the description of agronomical and morphological characteristics (FAO, 2004; Hong and Chau, 2004; Hoang, 2011). Thus, the tracing of longan varieties' history is confusing and complicated, because it is relaying on morphological observations, the features which are highly dependent on environmental conditions (Yonemoto et al., 2006).

Due to the high polymorphism level and the ability to discern between species, varieties and populations, molecular markers have been used as potential tools for the characterization of plants' gene pools (Oversná et al., 2002; Arif et al., 2010; Lammerts van Bueren et al., 2010). So far, several different molecular markers such

\footnotetext{
* Corresponding author: Faculty of Biotechnology, Ho Chi Minh City University of Food Industry, Ho Chi Minh City, Vietnam; e-mail: thehv@cntp.edu.vn
} 
as RAPD (Random Amplified Polymorphic DNA), AFLP (Amplified Fragment Length Polymorphism), SCoT (Start Codon Targeted Polymorphism), ISSR (Intersimple sequence repeat) have been applied for longan genetic diversity assessment (Lynch and Milligan, 1994; Chen and Liu, 2001, Cheng et al., 2015; Mariana et al., 2011). These markers have played an important role to distinguish, characterize, and elucidate germplasm origin and diversity. Among these markers, RAPD technique has been proven useful to detect the genetic diversity of different plants (Chen and Liu, 2001; Mei et al., 2014). RAPD, a technique where DNA bands are generated by using polymerase chain reaction (PCR) with random primers (short oligonucleotide sequences) can be applied for any organism as generated loci are theoretically unlimited (Lynch and Milligan, 1994). Several advantages such as low cost, short time of experiment, low DNA requirement, and no previous DNA sequence knowledge needed, predestine this method to be one of the first choices to access genetic variability.

RAPD markers have, however, a disadvantage - the method is highly sensitive to PCR reaction condition leading low reproducibility obtained by different laboratories. This drawback can be eliminated by SCAR markers, meanning the conversing of random binding RAPD primers to specific binding primers (Paran and Michelmore, 1993). SCAR markers allow the detection of single genetically defined loci and immediate scoring of results by the presence or absence of expected PCR bands. Thus, this method eliminates the need to sequence amplified products thereby researchers are able to decrease the cost and speed up analysis capacity (Yang et al., 2013). Consequently, SCAR markers offer the most practical method for screening numerous samples in a time and labor-saving manner due to its accuracy and cost efficiency (Kasai et al., 2000). In this study, two pairs of SCAR primers were developed and proven as high potential tool to identify 2 highly favorable longan cultivars, such as "Duong phen Hung Yen" and "Chin muon Hung Yen" in Vietnam.

\section{Material and methods}

\section{Plant material and DNA extraction}

Total of 11 longan leaf samples were collected from plants growing in different parts of Vietnam (Fig. 1 and Table 1). The samples were dried and preserved in silica gel until use. DNA from collected leaf samples was extracted with CTAB method (Cetyl Trimethyl Ammonium Bromide) as described by Madhou and coworkers (2013) and diluted to a final concentration of $10 \mathrm{ng} / \mu \mathrm{l}$, then stored at $-20^{\circ} \mathrm{C}$ until use.

\section{$R A P D$ analysis}

High quality DNA was subjected to RAPD reactions. The primers for PCR reactions were chosen from Yonemoto and coworkers (2006) and Mei and coworkers (2014) (Table 2) and purchased from IDT (Singapore). The RAPD reactions were performed in a total volume of $20 \mu \mathrm{l}$ containing $30 \mathrm{ng}$ DNA, $1 \times$ reaction buffer, $2 \mathrm{mM} \mathrm{MgCl}, 0.3 \mu \mathrm{M}$ of primer, $200 \mu \mathrm{M}$ of each dNTP, 1 unit of $T a q$ polymerase and sterile water to the final volume. PCR amplification was carried out in a SureCycler 8800 thermal cycler (Agilent, USA) as follows: initial denaturation at $95^{\circ} \mathrm{C}$ for 2 minutes; followed by 40 cycles of $30 \mathrm{~s}$ at $95^{\circ} \mathrm{C}, 30 \mathrm{~s}$ at $35^{\circ} \mathrm{C}, 54 \mathrm{~s}$ at $72^{\circ} \mathrm{C}$ and a final extension for $5 \mathrm{~min}$ at $72^{\circ} \mathrm{C}$. PCR amplification was then separated by electrophoresis in $1.5 \%$ agarose gel in $1 \times$ TAE buffer, stained with $0.5 \mu \mathrm{g} / \mathrm{ml}$ Gelred TM loading buffer and visualized using the Universal Hood Gel Doc system (Bio-Rad, USA). All reagents for PCR and electrophoresis were purchased from Biobasic (Canada) whereas Gelred for DNA staining was purchased from Biotium (USA).

\section{Cloning and sequencing of DNA fragments}

The RAPD reactions which generated amplified bands tightly linking to the specific genotypes were repeated for higher fidelity by using Pfu polymerase (Biobasic, Canada). PCR conditions were as mentioned above- for regular RAPD reactions. The appropriate bands were then excised from agarose gel and purified using DNA purification kit (Biolink, India) according to manufacturer's instructions and then sequenced following 2 sequencing strategies. Firstly, the purified RAPD fragments were sequenced using 3 '-extended oligonucleotide primers as described in Mitchelson and coworkers (1999) in an ABI automated sequencer (Applied Biosystems, USA) using a DyeDeoxy ${ }^{\mathrm{TM}}$ terminator cycle sequencing kit (Applied Biosystems, USA). The primers for direct sequencing are presented in Table S1 in supplementary materials.

Secondly, sequencing was performed following the protocol published by Yang and coworkers (2013). 


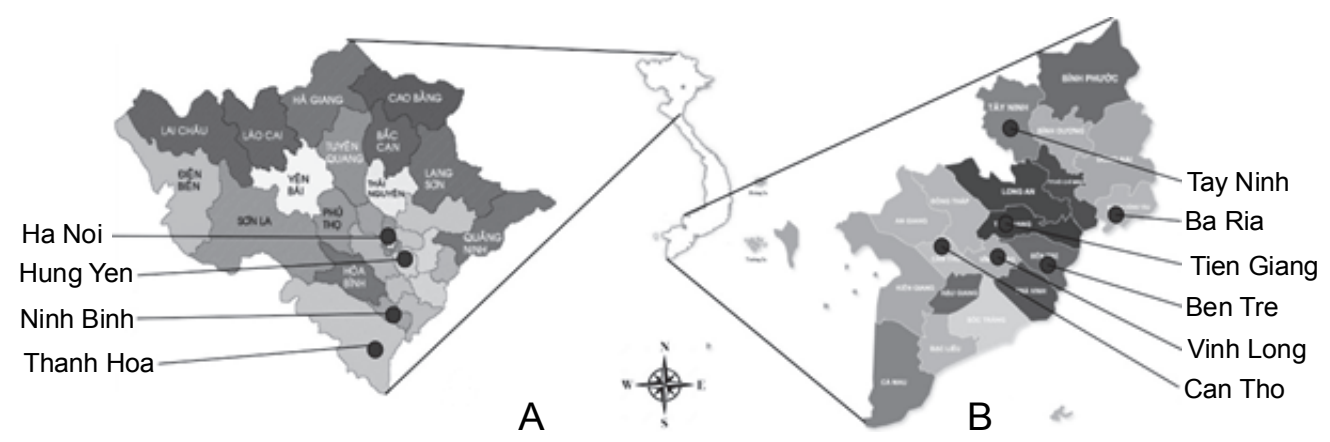

Fig. 1. The locations of longan plants used for samples in the present study

(black dots indicate sample collection sites; A and B representing Northern and Southern regions, respectively)

Table 1. The list of longan samples and its original locations used for genetic characterization in the present study

\begin{tabular}{c|c|c}
\hline Code & Accession name & Province \\
\hline DBR & Da bo Ba Ria & Ba Ria \\
\hline TBT & Thanh nhan Ben Tre & Ben Tre \\
\hline CHY & Chin muon Hung Yen & Hung Yen \\
\hline CTH & Chin muon Thanh Hoa & Thanh Hoa \\
\hline DHY & Duong phen Hung Yen & Hung Yen \\
\hline TTN & Tieu Tay Ninh & Tay Ninh \\
\hline XTG & Xuong Tien Giang & Tien Giang \\
\hline XVL & Xuong Vinh Long & Vinh Long \\
\hline TNB & Thoc Ninh Binh & Ninh Binh \\
\hline ICT & Idor Can Tho & Can Tho \\
\hline HHN & Huong Chi Ha Noi & Ha Noi \\
\hline
\end{tabular}

Briefly, the purified RAPD products were cloned using pJET1.2 cloning vector following the manufacturer's instructions (Thermo Scientific, USA). The plasmids containing RAPDs of interest were harvested from E. coli $\mathrm{DH} \alpha$ culture using miniprep isolation and purification kit (Life Technologies, USA) and subjected to DNA sequencing using vector-based primers (forward primer: 5'-CGACTCACTATAGGGAGAGCGGC-3', reverse primer: $5^{\prime}$ - AAGAACATCGATTTTCCATGGCAG-3'). The obtained DNA sequences were searched for homology in GenBank by BLAST tool from National Center for Biotechnology Information (NCBI, http://www.ncbi. nlm.nih.gov/BLAST/).

\section{Designing the SCAR primers}

SCAR primers were designed using 2 methods. Firstly using Primer3plus tool (www.bioinformatics.nl/ primer3plus). Secondly, following a method as described by Paran and Michelmore (1993), where from 8 to 12 bases were added to the original RAPD primers. The primer sequences, optimized PCR conditions and amplification lengths of these primers are shown in Table S2 in supplementary materials and Table 3 .

\section{Confirming the effectiveness of SCAR primers}

The polymorphisms of the newly designed SCAR primers were evaluated using 11 longan genotypes, as shown in Table 1. PCR reactions were prepared as follows: 30 ng DNA, $1 \times$ reaction buffer, $2 \mathrm{mM} \mathrm{MgCl}$, $0.3 \mu \mathrm{M}$ of each primer, $200 \mu \mathrm{M}$ of each dNTP, 1 unit of Taq polymerase (Biobasic, Canada) and sterile water to a final volume of $20 \mu \mathrm{l}$. PCR aliquots were then run in a SureCycler 8800 thermal cycler (Agilent, USA) with an initial pre-denaturation for $5 \mathrm{~min}$ at $95^{\circ} \mathrm{C}$ and followed by 35 cycles of denaturation at $95^{\circ} \mathrm{C}$ for $30 \mathrm{~s}$, annealing at for $30 \mathrm{~s}$ (annealing temperatures of primers is listed in Table 3), extension at $72^{\circ} \mathrm{C}$ for $40 \mathrm{~s}$. The final extension was performed at $72^{\circ} \mathrm{C}$ for $5 \mathrm{~min}$. PCR amplification products were analyzed using the same protocol as for RAPD analysis.

\section{Results and discussion}

As a result of RAPD reactions, all 20 primers tested (Table 1) resulted in amplification of a varying number of bands (from 3 to 14). The polymorphism among plant samples ranged from 0 to $77.78 \%$ (data not shown). Although RAPD results show high polymorphism among different longan accessions with band sizes ranging from $200 \mathrm{bp}$ to $2000 \mathrm{bp}$, in this study, the surveyed makers were not able to clearly identify any individual genotype. This is the common weakness of RAPD markers due to its dominant and unreliable features (Kumar and Guru- 
Table 2. The list of RARD primers used to analyze the genetic diversity of longan varieties grown in Vietnam

\begin{tabular}{c|c|c|c}
\hline $\begin{array}{c}\text { Primer } \\
\text { name }\end{array}$ & Primer sequence $\left(5^{\prime}-3^{\prime}\right)$ & Primer & Primer sequence $\left(5^{\prime}-3^{\prime}\right)$ \\
\hline A39a & CCTGAGGTAGCT & D12 & CTGGTCTCTGGG \\
\hline A58 & GTCATGCCTGGA & D29 & GACCCGGAACGA \\
\hline A62 & TCGTCCGGAGAT & D38 & AAGCTCGACGGG \\
\hline C11 & AGGTACGCCCGA & D41 & GAGACCCGTCGA \\
\hline C16 & CGCCCTGCAGTA & D50 & GACTCGCGGTCT \\
\hline C31 & GAGTTGCCCGGA & D53 & GCCGCGGAACTA \\
\hline C34 & TACCCAGGAGCG & D84 & AGACACACGGGC \\
\hline C59a & CGCGTTCGTGGA & F10a & CAGGCCGAAGTC \\
\hline C62a & CCATCCGCACGA & F44a & GGTGTCTTGCGG \\
\hline C82 & ATCGTCACCCCG & Z1 & CCGGTGCCTTCT \\
\hline
\end{tabular}

Table 3. SCAR primers designed by adding additional nucleotides to RAPD primers were used to distinguish different longan cultivars grown in Vietnam (the underlined sequences indicate the corresponding original RAPD primers)

\begin{tabular}{c|l|c|c}
\hline $\begin{array}{c}\text { SCAR } \\
\text { primer name }\end{array}$ & \multicolumn{1}{|c|}{ Sequence $\left(5^{\prime}-3^{\prime}\right)$} & $\begin{array}{c}\text { Product size } \\
{[\mathrm{bp}]}\end{array}$ & $\begin{array}{c}\mathrm{Tm} \\
{\left[{ }^{\circ} \mathrm{C}\right]}\end{array}$ \\
\hline DHY & $\begin{array}{l}\text { Fw - GACCCGGAACGAAATCATGAAAAA } \\
\mathrm{Rv}-\text { GACCCGGAACGATTTGTATGAGGT }\end{array}$ & 706 & 60 \\
\hline $\mathrm{CHY}$ & $\begin{array}{l}\mathrm{Fw}-\text { TCGTCCGGAGATAAAAAAAA } \\
\mathrm{Rv}-\text { TCGTCCGGAGATTCAGTTAGAC }\end{array}$ & 517 & 62 \\
\hline
\end{tabular}

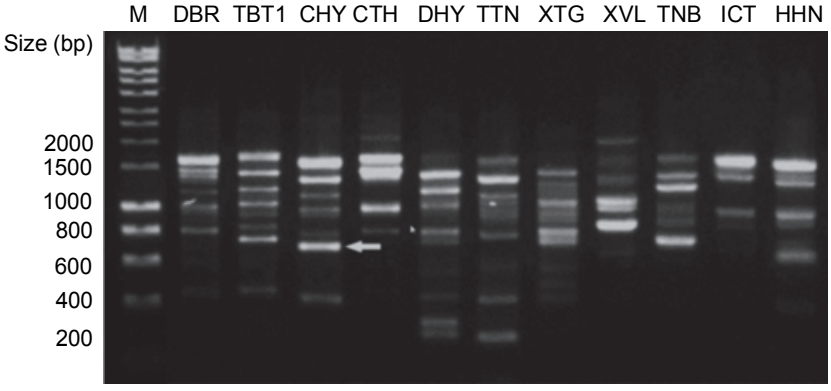

Fig. 2. The result of agarose gel electrophoresis showing RAPD amplification products of 11 longan genotypes, using D29 primer ( $\mathrm{M}$ - 1000 bp DNA ladder (Hyper ladder, Bioline, UK), white arrow indicates a candidate RAPD fragment for genotype identification)

subramanian, 2011). Another concern about RAPD is its reproducibility, where at least $15 \%$ of low signal bands should be excluded to increase the repeatability (Ali et al., 2004). Among several RAPD results, one band with size of about $700 \mathrm{bp}$ amplified by primer D29, dis-
M DBR TBT1 CHY CTH DHY TTN XTG XVL TNB ICT HHN

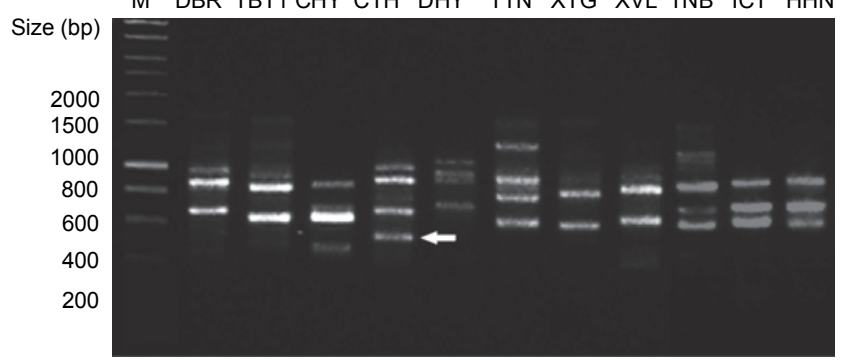

Fig. 3. The result of agarose gel electrophoresis showing RAPD amplification products of 11 longan genotypes C59a (M - 1000 bp DNA ladder (Hyper ladder, Bioline, UK), white arrow indicates a candidate RAPD fragment for genotype identification)

tinguishing "Duong phen Hung Yen/DHY" variety from remaining genotypes (Fig. 2) has been observed. Whereas, a band of approximate $500 \mathrm{bp}$ amplified using C59a primer was also considered as a candidate band to specifically identify "Chin muon Hung Yen/CHY" longan variety (Fig. 3). 


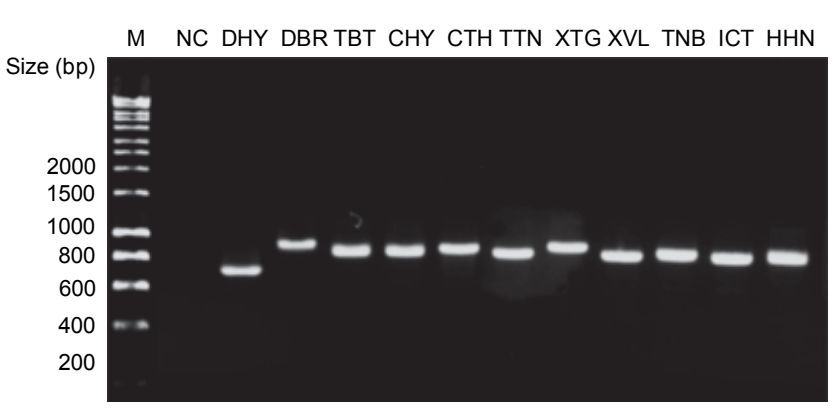

Fig. 4. PCR amplification of 11 longan genotypes in Vietnam by using DHY SCAR marker (M - 1000 bp DNA ladder; $\mathrm{NC}$ - negative control)

To further characterize 2 candidate markers, these 2 bands were excised, purified and sequenced. Initially, they were sequenced following a method reported by Mitchelson and coworkers (1999). From 2 original RAPD primers (D29 and C59a), 8 sequencing primers were obtained by adding one nucleotide (either A, T, G or C) at the $3^{\prime}$ ends (Table S1). Theoretically, the extended primer would bind to location of original RAPD primer and sequencing process would start from extended nucleotide. However the sequencing reactions were not successful from any of the primers tested (data not shown). We propose that since RAPD-amplified bands have 2 identical terminals, during sequencing reaction the signals from both terminals were mixed resulting in sequencing failure. In 2010, Jian and colleagues also found the limitation of this direct sequencing method. The authors reported that the factor contributing to the low success of this method could be the occurance of different nucleotides immediately internally to the primer-specified terminal (Jian et al., 2010), which could prevent the daily application of this method in practice.

Due to the unsuccessfulness of direct sequencing, these RAPD amplifications were inserted into pJET1.2 cloning vector and transformed into $E$. coli $\mathrm{DH} \alpha$. Then the purified cloning vector were harvested and subjected to sequence with specific primer using Sanger method. We successfully sequenced 2 candidate RAPD fragments indicated previously in Figure 2 and Figure 3 (the 2 sequences are presented in Supplementary data file S1). These 2 sequences were then deposited in GenBank of NCBI with accession numbers of MH061916 and MH061917 corresponding to sequences from "Duong phen Hung Yen/DPH" and "Chin muon Hung Yen/ CMH", respectively. This suggest that cloning-based sequencing is a preferred method to convert from RAPD

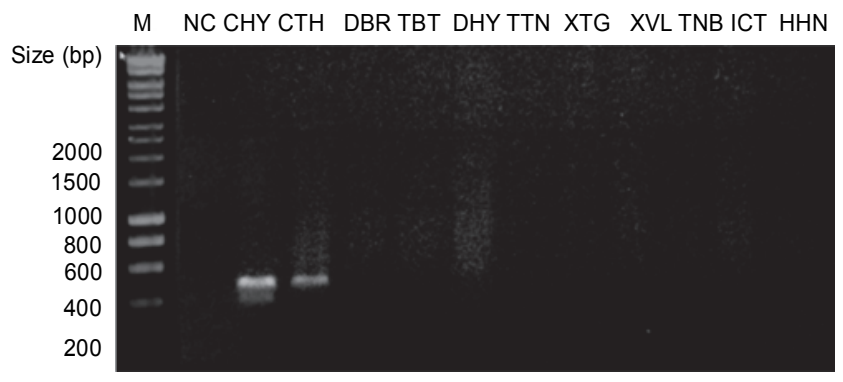

Fig. 5. PCR amplification of 11 longan genotypes in Vietnam by using CHY SCAR marker (M - 1000 bp DNA ladder; $\mathrm{NC}$ - negative control)

to SCAR marker and it has been used by previous research groups in various plant such as longan (Yang et al., 2013); Commiphora sp. (Sairkar et al., 2016). The obtained sequences were then searched for homology by BLAST analysis. The results showed no homological sequences among the existing accessions in GenBank, suggesting that these sequences have not been reported previously.

SCAR primers were initially designed based on internal parts obtained sequences (Table S2). However, these primers generated bands with the same amplification size for both sequences (data not shown). Thus, they were not able to differentiate among longan genotypes. Similar to our findings, considerable research groups also reported the difficulty in developing SCAR markers due to the formation of monomorphic products (Ohmori et al., 1996; Deng et al., 1997; Shen et al., 2011). However, several authors were successfulin using this type of primers in SCAR development like for Phyllanthus emblica (Dnyaneshwar et al., 2006); Nepenthes genus (Anuiwat et al., 2009), Lonicera japonica (Yang et al., 2014). This suggests that the success of internaldesigned primers could be an organism dependent feature. We then designed SCAR primers by adding 8-12 nucleotides followed corresponding RAPD markers shown in Table 3. The usefulness of such SCAR primers has been further evaluated. As expected, DHY primer was able to specifically amplify a sequence from "Duong phen Hung Yen/DHY" cultivar only; the remaining tested longan accessions showed unspecific amplification (Fig. 4). CHY primer was able to identify 2 "Chin muon" or "late ripening in Vietnamese" cultivars consisting of Chin Muon Hung Yen (CHY) and Chin muon Thanh Hoa (CTH), which were collected from Hung Yen and Thanh Hoa provinces, respectively (Fig. 5). 
SCAR markers are very useful in genetic analyses, however in this study we found that the success rate of discovering these markers in longan is relatively low. The most difficult point was the conversion of RAPD markers to SCAR markers ensuring the specificity. This could be explained because the SCAR primers designed from internal RAPD sequences are less polymorphic than those designed by adding nucleotides into initial RAPD primers and the polymorphism is likely only present in RAPD primers (Parasnis et al., 2000). Moreover, $\mathrm{GC}$ content is also another factor affecting the specificity of SCAR markers (Vanichanon et al., 2000), and SCAR primer design may require whole genome sequence information for effective development (Khan et al., 2008). This difficulty was expected as previously Cutler and coworkers (2006) found only 1 SCAR marker to differentiate 13 longan accessions after surveying 17 RAPD primers. Other groups also faced this problem when developing SCAR markers for litchi, which is a close relative of longan (Zhang et al., 2013).

\section{Conclusions}

The potential of RAPD markers has been proven-as a useful tool for genetic characterization and genetic diversity analyses which are important in plant science and agriculture. However, the conversion from RAPD to SCAR markers can improve the specificity and stability of amplifying targeted DNAs, which was successfully utilized for identifying different plant genotypes in a species. In this study we further confirmed that this marker is also efficient in identification different longan genotypes of different cultivars. In this research two pairs of SCAR primers which can be used to identify 2 varieties consisting of "Duong phen Hung Yen" and "Chin muon Hung Yen" have been successfully developed. The results of this project revealed that the conversion of RAPD markers into SCAR markers has a large potential for genetic identification of longan. Such markers could be potentially used for authentication of different plants.

\section{Acknowledgements}

Authors are thankful to Southeast Asian Regional Center for Graduate Study and Research in Agriculture (SEARCA) for financial support under Seed Fund for Research and Training (SFRT) programs. We also thank Faculty of Biotechnology, Ho Chi Minh City University of Food Industry for providing research facilities.

\section{References}

Ali B.A., Huang T.H, Quin D.N. Wang X.M. (2004) A review of random amplified polymorphic DNA (RAPD) markers in fish research. Rev. Fish. Biol. Fisher. 14: 443-453.

Arif I.A., Bakir M.A., Khan H.A., Al Farhan A.H., Al Homaidan A.A., Bahkai A.H., Al Sadoon M., Shobrak M. (2010) A brief review of molecular techniques to assess plant diversity. Int. J. Mol. Sci 11(5): 2079-2096.

Chen Y.Z., Liu C.M. (2001) Identification and analysis of 5 longan varieties by RAPD. China. Fru. 4: 28-29.

Cheng J., Long Y., Khan M.A., Wei C., Fu S., Fu J. (2015) Development and significance of RAPD-SCAR markers for identification of Litchi chinensis Sonn. by improved RAPD amplification and molecular cloning. Electr. J. Biotechnol. (18): 35-39.

Cutler R.W., Sitthiphrom S., Marha J., Anuntalabhochai S. (2006) Development of sequence-characterized DNA markers linkd to temperature insensitivity of fruit production in longan (Dimocarpus longan Lour.) cultivars. J. Agr. Cro. Sci. 193(1): 74-78.

Deng Z., Huang S., Xiao S., Gmitter F.G. (1997) Development and characterization of SCAR markers linkd to the citrus tristerza virus resistance gene from Poncirus trifoliate. Genome 40: 697-704.

Dnyaneshwar W., Preeti C., Kalpana J., Bhushan P. (2006) Development and application of RAPD-SCAR marker for identification of Phyllanthus emblica Linn. Biol. Phar. Bull. 29(11): 2313-2316.

FAO (2004) Fruits of Vietnam. Corporate document respository. Food and agriculture organization of the united nations, Regional Office for Asia and the Pacific, Bangkok, Thailand.

Hoang T.V.H. (2011) Development of fruit-tree in Dong Thap province: practices and solutions. Sci. J. Ho Chi Minh city Uni. Ped. 26: 74-79 (in vietnamese).

Hong B.M., Chau N.M. (2004) Longan production in Vietnam. [in:] Lychee, longan, rambutan and other Sapindaceae plants. Ed. N. Chomchalow, N. Sukhavibul, Proc. $2^{\text {nd }}$ Int. Symposium.

Jian L., Zhang Y.Z., Yu D.F, Zhu Z.Q. (2010) Molecular characterization of Cymbidium kanran cultivars based on extended random amplified polymorphic DNA (ERAPD) markers. Afr. J. Biotechnol. 9(32): 5084-5089.

Kasai K., Morikawa Y., Sorri V.A., Valkonen J.P.T, Gebhardt C., Watanabe K.N. (2000) Development of SCAR markers to the PVY resistance gene Ryadg based on a common feature of plant disease resistance genes. Genome (43): 1-8.

Khan S., Husaini A.M., Kiran U., Kamaluddin, Ram M., Abdin M.Z. (2008) SCAR markers for authentication of herbal drugs. Med. Aromat. Plant Sci. Biotech. 2(2): 79-85.

Kumar N.S., Gurusubramanian G. (2011) Ramdom amplified polymorphic DNA (RAPD) markers and its applifications. Sci. Vis. 11(3): 116-124.

Lammerts van Bueren E.T., Backes G., de Vriend H., Østergård H. (2010) The role of molecular markers and marker 
assisted selection in breeding for organic agriculture. Euphytica 175(1): 51-64.

Lynch M., Milligan B.G. (1994) Analysis of population genetic structure with RAPD marker. Mol. Ecol. (3): 91-99.

Madhou M., Normand F., Bahorun R., Hormaza J.I. (2013) Fingerprinting and analysis of genetic diversity of litchi (Litchi chinensis Sonn.) accessions from different germplasm collections using microsatellite markers. Tree. Genet. Genomes 9: 387-396.

Mariana B.D., Sugiyatno A., Supriyanto A. (2011) Genetic diversity of local accessions of Dimocarpus longan revealed by ISSR marker. Bull. Plasma Nutfah 17(1): 25-29.

Mei Z.Q., Fu S.Y., Yu H.Q., Yang L.Q., Duan C.G., Liu X.Y., Gong S., Fu J.J. (2014) Genetic characterization and authentication of Dimocarpus longan Lour. Using an improved RAPD technique. Genet. Mol. Res. 13(1): 1447-1455.

Mitchelson K.R., Drenth J., Duong H., Chaparro J.X. (1999) Direct sequencing of RAPD fragments using 3'-extended oligonucleotide primers and dye terminator cycle-sequencing. Nucl. Acids Res. 27(19): e28.

Ohmori T., Murata M., Motoyoshi F. (1996) Molecular characterization of $R A P D$ and $S C A R$ markers linked to the Tm-1 locus in tomato. Theor. Appl. Genet. 92: 151-156.

Oversná J., Poláková K., Leisová L. (2002) DNA analyses and their applications in plant breeding. Czech J. Genet. Plant Breed. 38(1): 29-40.

Paran I., Michelmore R.W. (1993) Development of reliable PCR-based markers linked to downy mildew resistance genes in lettuce. Theor. Appl. Genet. 85: 985-993.

Parasnis A.S., Gupta V.S., Tamhankar S.A., Ranjekar P.K. (2000) A highly reliable sex diagnostic PCR assay for massscreening of Papaya seedlings. Mol. Breed. (6): 337-344.
Sairkar P.K., Sharma A., Shukla N.P. (2016) SCAR marker for identification and discrimination of Commbiphora wightii and C. myrrha. Mol. Biol. Int. Article ID 1482796. http:// dx.doi.org/10.1155/2016/1482796.

Shen A., Li H., Wang K., Ding H. Zhang X., Fan L., Jiang B. (2011) Sequence characterized amplified region (SCAR) markers-based rapid molecular typing and identification of Cunninghamia laceolata. Afr. J. Biotechnol. 10(82): 19066-19074.

Tien Giang Promotion Agency (2017) Export-import information of fruit market. Retrieved from ttxddt.tiengian.gov.vn on February 28, 2018 (in vietnamese).

Vanichanon A., Blake N.K., Martin J.M., Talbert L.E. (2000) Properties of sequence-tagged-site primer sets influencing repeatability. Genome 43: 47-52.

Yang L., Fu S., Khan M.A., Zeng W., Fu J. (2013) Molecular cloning and development of RAPD-SCAR markers for Dimocarpus longan variety authentication. Springerplus 2: 501.

Yang L., Khan M.A., Mei Z., Yang M., Zhang T., Wei C., Yang W., Zhu L., Long Y., Fu J. (2014) Development of RAPD$S C A R$ markers for Lonicera japonica (Caprifolicaceae) variety authentication by improved $R A P D$ and DNA cloning. Rev. Biol. Trop. 62(4): 1649-1657.

Yonemoto Y., Chowdury A.K., Kato H., Macha M.M. (2006) Cultivar identification and their genetic relationships in Dimocarpus longan subspecies based on RAPD markers. Sci. Hortic. 109: 147-152.

Zhang W., Yin L., Wei S., Deng Z., Yi J., Wu R., Chen Q. (2013) RAPD marker conversion into a SCAR marker for rapid identification of Johnsongrass [Sorghum halepense (L.) Pers.J. Not. Bot. Horti. Agrobot. 41(1): 306-312. 